\title{
Research Article \\ Effect of Vacancy Defects on the Electronic Structure and Optical Properties of GaN
}

\author{
Lili Cai and Cuiju Feng \\ Foundation Department, North China Institute of Science and Technology, Beijing 101601, China \\ Correspondence should be addressed to Lili Cai; lily2004c@163.com
}

Received 20 November 2016; Accepted 12 January 2017; Published 12 February 2017

Academic Editor: Yongxing Zhang

Copyright (C) 2017 Lili Cai and Cuiju Feng. This is an open access article distributed under the Creative Commons Attribution License, which permits unrestricted use, distribution, and reproduction in any medium, provided the original work is properly cited.

\begin{abstract}
The effect of gallium vacancy $\left(V_{\mathrm{Ga}}\right)$ and nitrogen vacancy $\left(V_{\mathrm{N}}\right)$ defects on the electronic structure and optical properties of GaN using the generalized gradient approximation method within the density functional theory were investigated. The results show that the band gap increases in $\mathrm{GaN}$ with vacancy defects. Crystal parameters decrease in GaN with nitrogen vacancy $\left(\mathrm{GaN}: V_{\mathrm{N}}\right)$ and increase in $\mathrm{GaN}$ with gallium vacancy $\left(\mathrm{GaN}: V_{\mathrm{Ga}}\right)$. The Ga vacancy introduces defect levels at the top of the valence band, and the defect levels are contributed by $\mathrm{N} 2 p$ electron states. In addition, the energy band shifts to lower energy in GaN: $V_{\mathrm{N}}$ and moves to higher energy in $\mathrm{GaN}: V_{\mathrm{Ga}}$. The level splitting is observed in the $\mathrm{N} 2 \mathrm{p}$ states of GaN: $V_{\mathrm{N}}$ and Ga3d states of GaN: $V_{\mathrm{Ga}}$. New peaks appear in lower energy region of imaginary dielectric function in $\mathrm{GaN}: V_{\mathrm{N}}$ and $\mathrm{GaN}: V_{\mathrm{Ga}}$. The main peak moves to higher energy slightly and the intensity decreases.
\end{abstract}

\section{Introduction}

$\mathrm{GaN}$ is so called third generation semiconductor materials with wide band gap. With the rapid developments of semiconductor technology, it has become the research focus in semiconductor materials in past decades. The band gap of $\mathrm{GaN}$ is $3.39 \mathrm{eV}$ at room temperature. Particularly, the GaNbased materials have the advantages of wide band gap, fast electron drift velocity, high temperature resistance, high pressure tolerance, and antiradiation [1]. So they have attracted much attention in the preparation of short wavelength luminescence device and high power microwave device. $\mathrm{GaN}$ is the core material and basic device of the new semiconductor optoelectronics industry. The defects and impurities in GaN have a great influence on the electron transport and optical properties of materials. Simulation is a good method to study defects in semiconductor materials except experiment. There is a lot of research on $\mathrm{GaN}$ used experiment or theoretical calculation [2-4]. Neugebauer and Van De Walle calculated the electronic structure and atomic structure of the intrinsic defects of $\mathrm{GaN}$ by first principles [5]. Boguslawski et al. simulated the intrinsic defects of $\mathrm{GaN}$ using ab initio molecular dynamics [6]. Kang et al. calculated the defects in
GaN epitaxial layer by first principles. It is believed that C, $\mathrm{O}$ impurity and intrinsic defects may be the sources of yellow and blue light [7]. Shen and Kang studied the intrinsic defects of six-party $\mathrm{GaN}$ and the electronic structure of $\mathrm{C}$ and $\mathrm{O}$ using local density functional theory [8]. These methods are based on first principles to calculate the formation energy of $\mathrm{GaN}$ and the electronic structure of $\mathrm{C}$ and $\mathrm{O}$. In this paper, we studied the effect of $V_{\mathrm{Ga}}$ and $V_{\mathrm{N}}$ on the electronic structure and optical properties of $\mathrm{GaN}$ using first principles.

\section{Model and Calculation}

Pure $\mathrm{GaN}$ is hexahedron wurtzite structure. It belongs to P63mc space group. Its symmetry is $\mathrm{C}_{6 \mathrm{v}-4}$. The lattice constants are $a=b=0.3189 \mathrm{~nm}, c=0.5185 \mathrm{~nm}, \alpha=\beta=90^{\circ}$, and $\gamma=120^{\circ} . c / a$ is 1.626 . It is slightly smaller than the ideal hexagonal close packed structure of 1.633. Figure 1(a) shows supercell model of $\mathrm{GaN}$ which contains $16 \mathrm{Ga}$ atoms and $16 \mathrm{~N}$ atoms. The coordinate of $V_{\mathrm{Ga}}$ is $(0.66667,0.33333$, $0.49926)$ and that of $V_{\mathrm{N}}$ is $(0.33333,0.66667,0.4375)$ (as shown in Figures 1(b) and 1(c)). The calculation is based on the CASTEP (Serial Total Energy Package Cambridge) of Material Studio 7.0 in this paper. The interaction between 


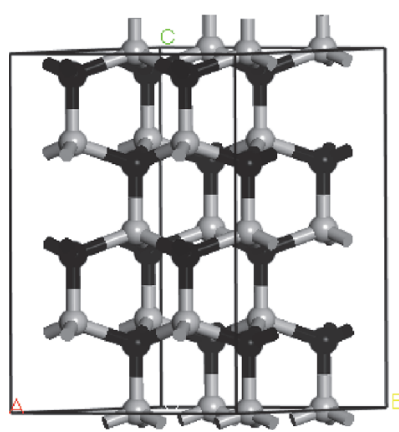

(a)

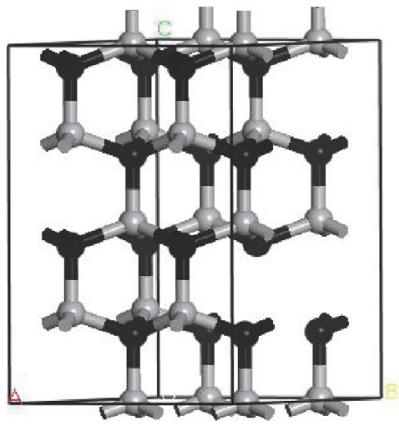

(b)

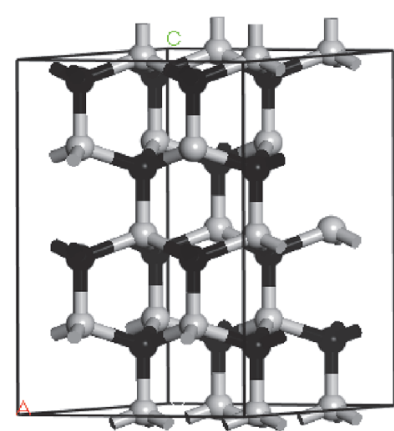

(c)

Figure 1: (a) $2 \times 2 \times 2 \mathrm{GaN}$ supercell; black stands for $\mathrm{N}$ atoms and grey stands for Ga atoms, (b) GaN with Ga vacancy (GaN: $V_{\mathrm{Ga}}$ ), and (c) $\mathrm{GaN}$ with $\mathrm{N}$ vacancy $\left(\mathrm{GaN}: V_{\mathrm{N}}\right)$.

TABLE 1: Lattice parameters of GaN after geometry optimization.

\begin{tabular}{lccc}
\hline & $a / \mathrm{nm}$ & $c / \mathrm{nm}$ & $c / a$ \\
\hline $\mathrm{GaN}(\operatorname{Exp})$ & 0.3189 & 0.5185 & 1.626 \\
$\mathrm{GaN}$ & 0.3226 & 0.5253 & 1.628 \\
$\mathrm{GaN}: V_{\mathrm{N}}$ & 0.3229 & 0.5241 & 1.623 \\
$\mathrm{GaN}: V_{\mathrm{Ga}}$ & 0.3224 & 0.5263 & 1.632 \\
\hline
\end{tabular}

electrons and ions is described by means of plane wave pseudopotential method (PWP) with generalized gradient approximation (GGA). Energy cut-off of plane wave is $360 \mathrm{eV}$. The convergent accuracy is $2 \times 10^{-6} \mathrm{eV} /$ atom during iteration. The maximum force is $0.03 \mathrm{eV} / \mathrm{A}$ and the maximum stress is $0.05 \mathrm{GPa}$. Monkhorst-Pack is used to calculate the total energy and charge density in the Brillouin zone. $K$-point set is $4 \times 4 \times 2$. Fast Fourier transform (FFT) grid is $40 \times 40 \times 72$. All the calculations are carried out in the reciprocal space. The valency electrons in calculation are Ga: $3 d^{10} 4 s^{2} 4 p^{1}, N: 2 s^{2} 2 p^{3}$.

\section{Results and Discussions}

3.1. Structure and Properties. Table 1 shows the lattice constants of pure and vacancy defects GaN after optimization. $c / a$ is 1.628 in pure $\mathrm{GaN}$, which is close to others' [9]. The error is only $0.12 \%$ comparing with experimental results 1.626. The lattice constant $c$ decreases in $\mathrm{GaN}: V_{\mathrm{N}}$ and increases in GaN: $V_{\mathrm{Ga}}$. However, vacancy type has a little effect on the lattice constant $a$. This result is similar to the literature [10]. The result indicates that $V_{\mathrm{N}}$ causes a larger relaxation in [1000] direction, but $V_{\mathrm{Ga}}$ has larger relaxation in [0001] direction.

3.2. Band Structure and Density of States. Figure 2 shows the band structure of the pure GaN and vacancy defects GaN. The valence band maximum and conduction band minimum of pure $\mathrm{GaN}$ are located in the $\mathrm{G}$ point of Brillouin zone. The results indicate that $\mathrm{GaN}$ is a direct wide band gap semiconductor material with energy gap of $1.66 \mathrm{eV}$. This result is consistent with Du et al.s [11]. But it is significantly different from the experimental value of $3.39 \mathrm{eV}$. This is due to the use of the generalized gradient approximation (GGA) and ignoring the correlation of excited state electrons. It has no effect on result discussion [12]. It can be seen from Figure 2 that the numbers of valence band and conduction band significantly increase in $\mathrm{GaN}: V_{\mathrm{Ga}}$ and $\mathrm{GaN}: V_{\mathrm{N}}$ and the band gap is wider than that of the pure GaN. The band gaps of GaN: $V_{N}$ and $\mathrm{GaN}: V_{\mathrm{Ga}}$ increase to $2.30 \mathrm{eV}$ and $1.97 \mathrm{eV}$, respectively. $\mathrm{GaN}$ becomes an indirect band gap semiconductor because of $V_{\mathrm{N}}$. Three acceptor levels are introduced at $0.67 \mathrm{eV}$ near the top of valence band in GaN: $V_{\mathrm{Ga}}$. The Fermi level $\left(E_{f}\right)$ moves to lower energy regions obviously. The Fermi level occupies the part of valence band maximum. Electrons in valence band can jump to this level. Holes can occur in the valence band. So Ga vacancy is acceptor defect in GaN.

The total density of states (TDOS) of pure GaN and vacancy defect GaN is shown in Figure 3. It can be seen from Figure 3 that the valence band of pure GaN is mainly composed of two group bands. The valence band above is mainly from Ga3d and $\mathrm{N} 2 s$ states. It ranges from $-15.9 \mathrm{eV}$ to $-10.4 \mathrm{eV}$. The peak of Ga3d state appears at $-13.5 \mathrm{eV}$ and forms a strong local density of states. There are no obvious interactions with other valence bands. The valence band below is from $-6.9 \mathrm{eV}$ to $0 \mathrm{eV}$. It is mainly composed of $\mathrm{N} 2 \mathrm{p}$ state which determines the position of valence band. The conduction band is from $3.0 \mathrm{eV}$ to $7.7 \mathrm{eV}$. It mainly consists of Ga4s and Ga4P states and a few of N2p states. N2s states have less contribution to the valence and conduction band. Near the Fermi level, the variation of DOS at the bottom of the conduction band is relatively smooth. The electron has a small effective mass and high mobility in the conduction band [13]. Figure 3(b) shows that $V_{\mathrm{N}}$ has great effect on the TDOS of GaN. The valence and conduction band of GaN: $V_{N}$ move to lower energy regions obviously. The conduction band minimum shifts to lower energy and occupies the Fermi level. A shallow donor level is produced at the bottom of the conduction band and its width is about $0.8 \mathrm{eV}$. GaN presents n-type conductivity. The valence band of $\mathrm{GaN}: V_{\mathrm{Ga}}$ moves to higher energy regions and the Fermi level jumps into the valence band. But the movement is relatively small. The Fermi level occupies part of the valence band maximum. The electrons in the valence band jump to this level and holes are produced in the valence band. $V_{\mathrm{Ga}}$ is the acceptor 


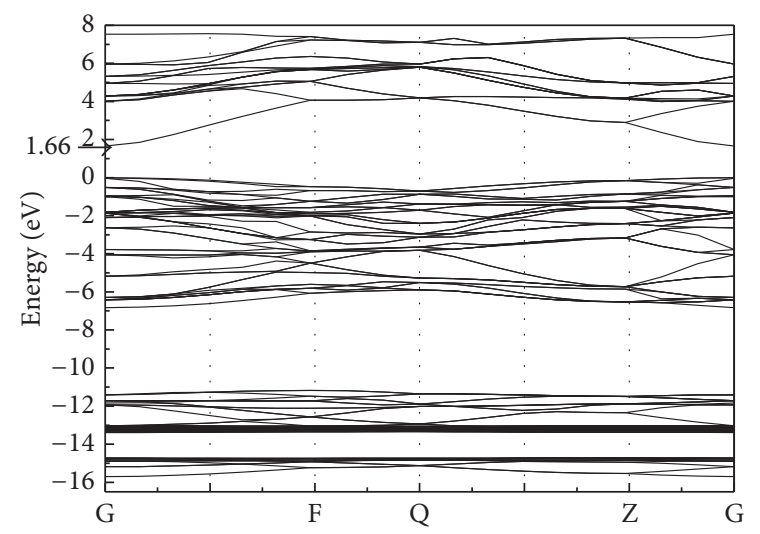

(a)

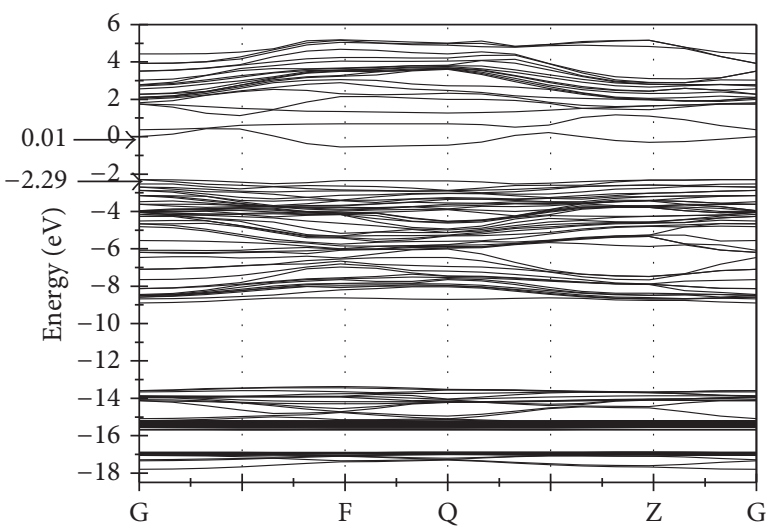

(b)

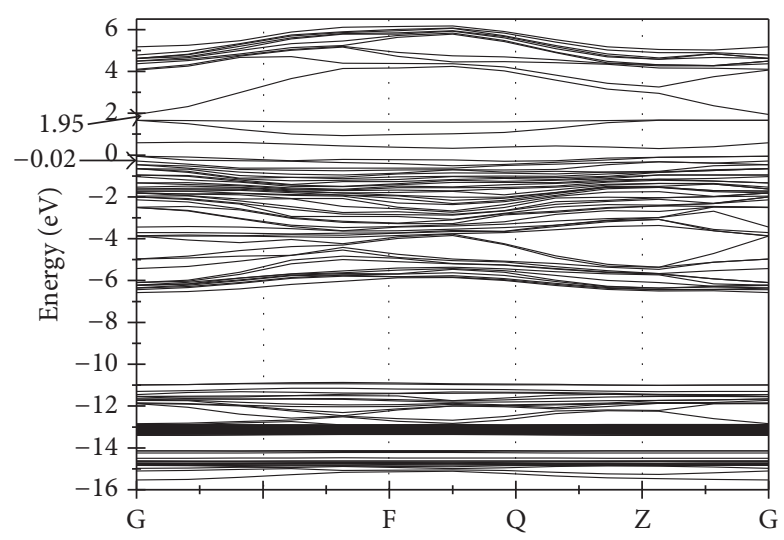

(c)

Figure 2: Band structure of pure $\mathrm{GaN}(\mathrm{a}), \mathrm{GaN}: V_{\mathrm{N}}(\mathrm{b})$, and $\mathrm{GaN}: V_{\mathrm{Ga}}$ (c).

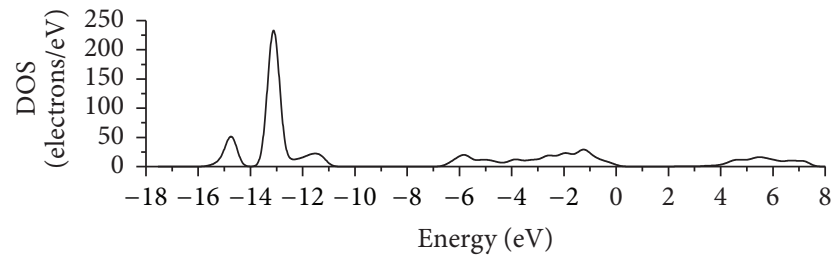

(a) GaN

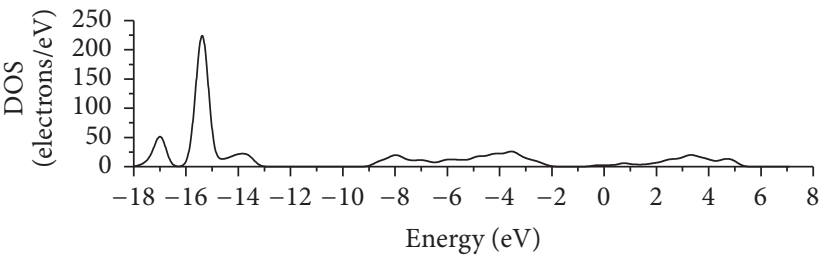

(b) GaN: $V_{\mathrm{N}}$

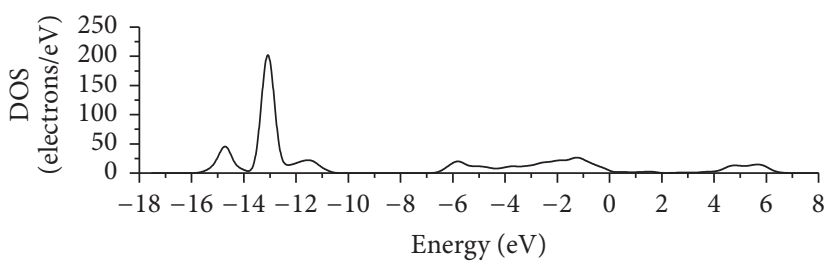

(c) GaN: $V_{\mathrm{Ga}}$

FIGURE 3: Total density of state of pure and vacancy GaN.

defect for GaN. The peak of DOS decreases significantly in the valence band of $\mathrm{GaN}: V_{\mathrm{N}}$ and $\mathrm{GaN}: V_{\mathrm{Ga}}$. The peak of $\mathrm{GaN}: V_{\mathrm{Ga}}$ decreases more obviously. The density of states in $\mathrm{GaN}: V_{\mathrm{N}}$ shows a significant decline near the Fermi level. The conductivity is proportional to the density of states at the Fermi level. So we can confirm that $V_{\mathrm{N}}$ can reduce the conductivity of GaN. It is also found that vacancy causes band gap increase from Figure 3.

Figures 4 and 5 show the partial density of states of $\mathrm{Ga}$ and $\mathrm{N}$ atoms, respectively. The bottom of the conduction band is mainly determined by the Ga4s electron states in the pure and vacancy defects GaN. It can be seen from Figure 5(c) that 


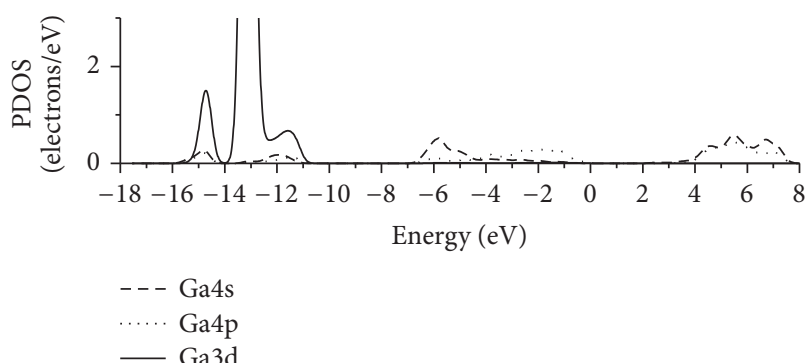

(a) $\mathrm{GaN}$

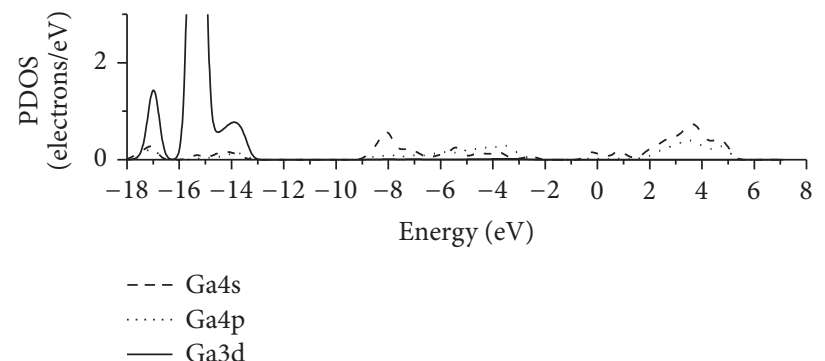

(b) $\mathrm{GaN}: V_{\mathrm{N}}$

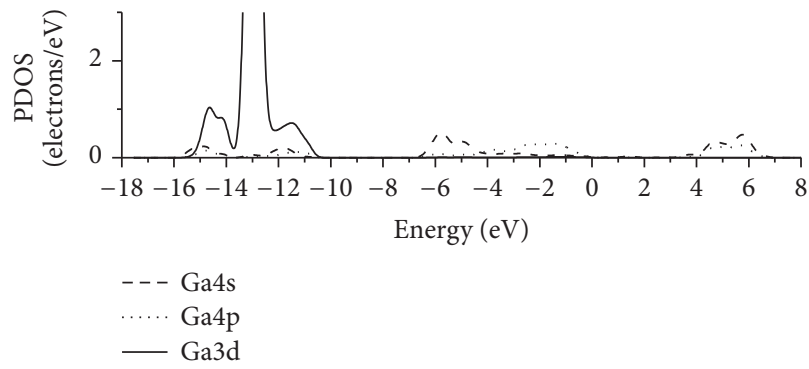

(c) $\mathrm{GaN}: V_{\mathrm{Ga}}$

FIgUre 4: Partial density of state of Ga atom.

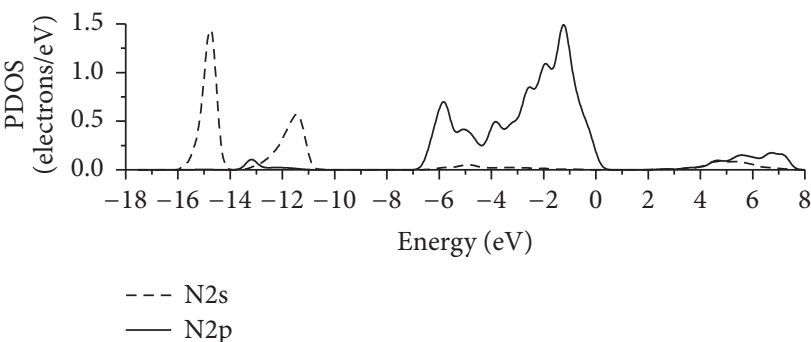

(a) $\mathrm{GaN}$

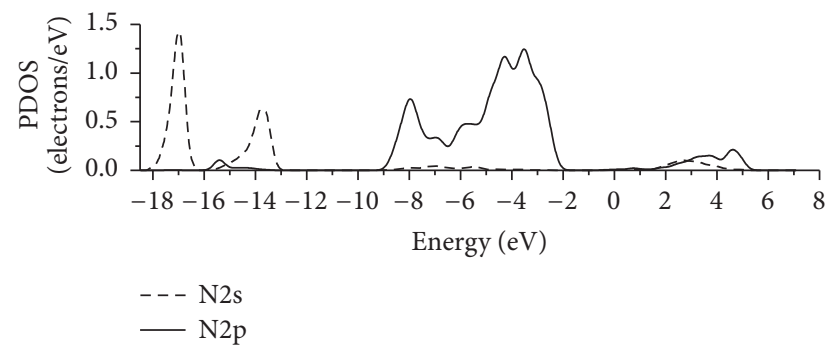

(b) $\mathrm{GaN}: V_{\mathrm{N}}$

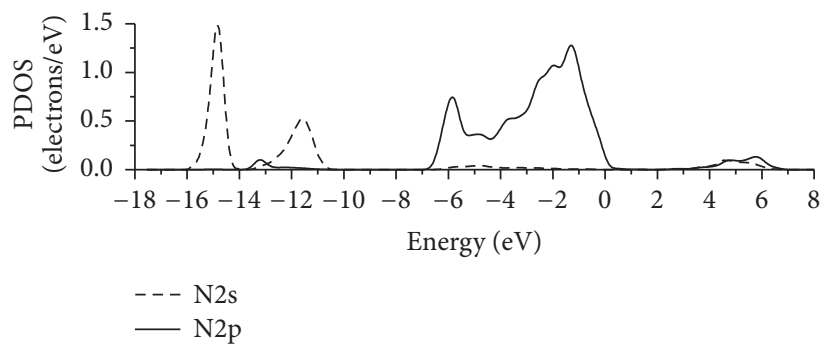

(c) $\mathrm{GaN}: V_{\mathrm{Ga}}$

FIGURE 5: Partial density of state of $\mathrm{N}$ atom.

the acceptor level in the valence band maximum of $\mathrm{GaN}: V_{\mathrm{Ga}}$ is mainly contributed by $\mathrm{N} 2 \mathrm{p}$ electron states. $\mathrm{N} 2 \mathrm{p}$ electrons cross Fermi level to occupy a higher energy level. Unfilled electron states are formed at the top of valence band. GaN presents p-type conductivity. Figure 5(b) shows that N2p states of $\mathrm{GaN}: V_{\mathrm{N}}$ form level splitting at $-4 \mathrm{eV}$ and $4 \mathrm{eV}$. This may be the S-like and P-like local states caused by $V_{\mathrm{N}}[14]$. Slike states are in the valence band and P-like states are in the conduction band. Instead of level splitting, a greater level is formed in $\mathrm{GaN}: V_{\mathrm{Ga}}$ as shown in Figure 5(c). We can see from
Figure 4(c) that Ga3d states of $\mathrm{GaN}: V_{\mathrm{Ga}}$ form two split levels at about $-15 \mathrm{eV}$. The two levels are $0.8 \mathrm{eV}$ apart. It is believed that the main reason for the level splitting is the quantum effect interactions between atoms. The absence of $\mathrm{Ga}$ atoms destroys the periodic structure of the perfect GaN. N atoms superposition and hybridization with Ga cause Ga3d states split below valence band in $\mathrm{GaN}: V_{\mathrm{Ga}}$.

3.3. Influence of Defects on the Optical Properties of GaN. Adiabatic approximation and single electron approximation 
are usually used in the interaction between light and solid. The phonon indirect transition can be neglected using the single electron approximation. Only electronic excitation is considered [15]. Complex permittivity is $\varepsilon(\omega)=\varepsilon_{1}(\omega)+$ $i \varepsilon_{2}(\omega)$ and complex refractive index is $N(\omega)=n(\omega)+i k(\omega)$, which can describe the linear response of the system to the photoelectric field in the wavelet vector. The imaginary part of the dielectric constant can be deduced according to the direct transition probability and dispersion relationship [16]:

$$
\begin{aligned}
& \varepsilon_{2}(\omega)=\frac{C}{\omega^{2}} \sum_{C, V} \int_{B Z} \frac{2}{(2 \pi)^{3}}\left|M_{C V}(K)\right|^{2} \\
& \times \delta\left(E_{k C}-E_{k V}-h \omega\right) d^{3} K .
\end{aligned}
$$

The subscripts of $C$ and $V$ are the conduction band and valence band, respectively. $\mathrm{BZ}$ is the first Brillouin zone. $K$ is the electron wave vector. $M_{C V}(K)$ is the transition matrix element. $E_{K C}$ and $E_{K V}$ are the intrinsic levels of the conduction band and valence band. Formula (1) provides the basic theoretical basis for the analysis of the structure and optical properties of crystal. It reflects the luminescence mechanism of the electronic transitions between levels. The spectra are generated by electronic transitions between levels. Each dielectric peak can be explained by band structure and density of states [17].

The imaginary dielectric functions of the pure GaN and vacancy defect $\mathrm{GaN}$ are given in Figure 6. It can be seen from Figure 6 that a very strong peak appears in the range of 5$10 \mathrm{eV}$. The principal peak of pure $\mathrm{GaN}$ is located at $5.89 \mathrm{eV}$. It is supposed to be caused by the Ga3d or N2p electrons transition to lower levels. The principal peak in GaN: $V_{N}$ and $\mathrm{GaN}: V_{\mathrm{Ga}}$ shifts to higher energy regions slightly. The spectrum shows blueshift. The intensity of the principal peak is weakened. This is due to the lattice relaxation induced by vacancy in $\mathrm{GaN}$. There is no dielectric peak in $0-2 \mathrm{eV}$ in pure GaN. But dielectric peak is found at about $0.82 \mathrm{eV}$ in GaN: $V_{\mathrm{N}}$ and $\mathrm{GaN}: V_{\mathrm{Ga}}$. And the peak in $\mathrm{GaN}: V_{\mathrm{N}}$ is stronger. This peak comes from the transition between valence band and conduction band. The peak at $2.83 \mathrm{eV}$ in the pure $\mathrm{GaN}$ should be $\mathrm{N} 2 \mathrm{p}$ electrons transition from valence band maximum to conduction band. There is deviation between the peak and energy gap, which is probably related to the relaxation effect of N2p electrons transition. The peak becomes a rising edge and shifts to higher energy regions in $\mathrm{GaN}: V_{\mathrm{N}}$. It is consistent with the band gap increase in $G a N: V_{N}$. Ga vacancy will introduce a deep acceptor level near the Fermi level. So the peak at $2.83 \mathrm{eV}$ in $\mathrm{GaN}: V_{\mathrm{Ga}}$ is considered to be the transition between the conduction band and the deep acceptor level. Ga vacancy was once believed to be the source of yellow light $(2.2-2.8 \mathrm{eV})[18]$. The peaks are the same at $10.97 \mathrm{eV}$ and $18.22 \mathrm{eV}$ in pure and vacancy GaN. The peak at $10.97 \mathrm{eV}$ may be the Ga3d or N2s electrons transition between the valence band above and below. The peak at $18.22 \mathrm{eV}$ should be the Ga3d transition between the valence band below and the conduction band. Most electrons in valence band below jump to valence band above in the first and then transit from valence band above to conduction band. There

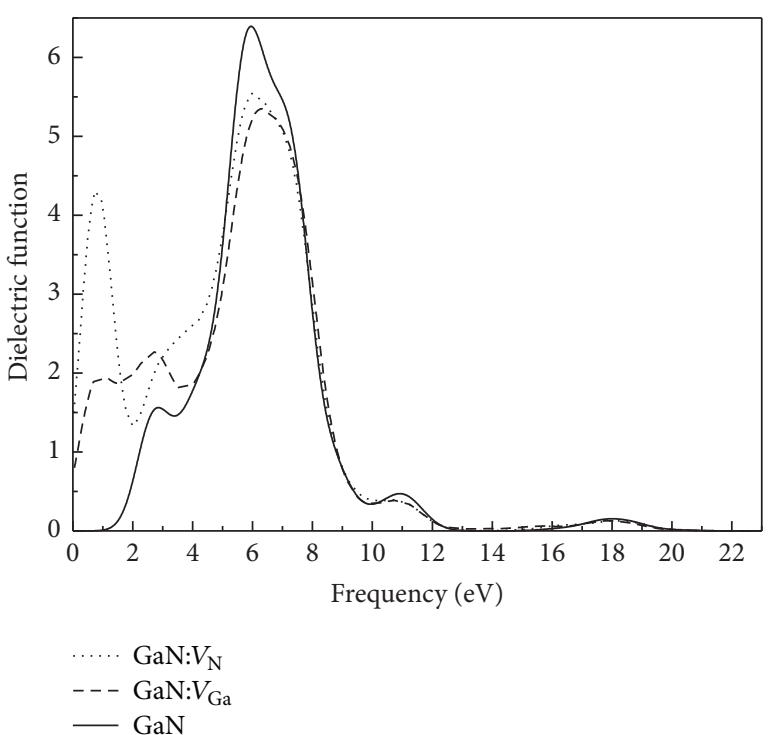

FIgURE 6: The imaginary part of the complex dielectric function of the pure and vacancy defects GaN.

are few electrons transitioning from valence band below to conduction band directly. So the peak is very weak.

\section{Conclusions}

The electronic structure and optical properties of pure GaN and $\mathrm{GaN}: V_{\mathrm{N}}, \mathrm{GaN}: V_{\mathrm{Ga}}$ have been investigated by means of plane wave pseudopotential method (PWP) with generalized gradient approximation (GGA). The vacancy defects make lattice constant change and band gap increase. The valence and conduction band shift to lower energy regions obviously in $\mathrm{GaN}: V_{\mathrm{N}}$ compared with pure GaN. The valence band moves to higher energy regions in $\mathrm{GaN}: V_{\mathrm{Ga}}$. Vacancy makes the density of state decrease obviously in the valence band below of $\mathrm{GaN}$. The peak decreases more in the $\mathrm{GaN}: \mathrm{V}_{\mathrm{Ga}}$. Ga4s electron states decide the conduction band minimum and the valence band maximum is determined by N2p states in pure $\mathrm{GaN}, \mathrm{GaN}: V_{\mathrm{N}}$, and $\mathrm{GaN}: V_{\mathrm{Ga}}$. Optical properties are induced by vacancy defects. New dielectric peaks appear in lower energy regions in $\mathrm{GaN}: V_{\mathrm{N}}$ and $\mathrm{GaN}: V_{\mathrm{Ga}}$. The peaks are produced by electronic transitions from the valence band to the conduction band. Compared with pure $\mathrm{GaN}$, the principal peaks in $\mathrm{GaN}: V_{\mathrm{N}}$ and $\mathrm{GaN}: V_{\mathrm{Ga}}$ shift to higher energy region slightly. At the same time, the intensity is weakened and blueshift happened.

\section{Competing Interests}

The authors declare that they have no competing interests.

\section{Acknowledgments}

This work was supported by the Langfang City Key Technology Research and Development Program of China (no. 
2016011054) and the Fundamental Research Funds for the Central Universities of China (no. 3142016010).

\section{References}

[1] A. Majid, J. Iqbal, and A. Ali, "Structural, optical and magnetic properties of Ce-GaN based diluted magnetic semiconductor," Journal of Superconductivity and Novel Magnetism, vol. 24, no. 1-2, pp. 585-590, 2011.

[2] Q. Q. Li, Q. Y. Hao, Y. Li, and G. Liu, "First principle study of Ce doping and related complexes in GaN," Computational Materials Science, vol. 72, pp. 32-37, 2013.

[3] J. K. Shen and F. W. Huang, "Improved conversion efficiency of GaN-based solar cells with Mn-doped absorption layer," Applied Physics Letters, vol. 103, no. 6, Article ID 063906, 4 pages, 2013.

[4] A. Majid, A. Dar, and J. J. Zhu, "Cerium induced ferromagnetic exchange interactions in GaN," Journal of Magnetism and Magnetic Materials, vol. 374, pp. 676-679, 2015.

[5] J. Neugebauer and C. G. Van De Walle, "Atomic geometry and electronic structure of native defects in GaN," Physical Review $B$, vol. 50, no. 11, pp. 8067-8070, 1994.

[6] P. Boguslawski, E. L. Briggs, and J. Bernholc, "Native defects in gallium nitride," Physical Review B, vol. 51, no. 23, pp. 1725517258, 1995.

[7] J. Kang, D. Cai, X. Xiao, Y. Shen, and B. Shen, "Defects in III nitrides epilayers," Research and Progress of Solid State Electronics, vol. 22, no. 4, pp. 375-380, 2002.

[8] Y.-W. Shen and J.-Y. Kang, "Ab initio calculation of the electronic structure of carbon and oxygen impurities in GaN," Wuli Xuebao/Acta Physica Sinica, vol. 51, no. 3, pp. 645-648, 2002.

[9] G. Y. Gao, K. L. Yao, Z. L. Liu, Y. L. Li, Y. C. Li, and Q. M. Liu, "Ab initio pseudopotential studies of the pressure dependences of structural, electronic and optical properties for GaN," Solid State Communications, vol. 138, no. 10-11, pp. 494-497, 2006.

[10] W. E. Packard, J. D. Dow, K. Doverspike, R. Kaplan, and R. Nicolaides, "Vacancy structures on the GaN(0001) surface," Journal of Materials Research, vol. 12, no. 3, pp. 646-650, 1997.

[11] Y.-J. Du, B.-K. Chang, J.-J. Zhang, B. Li, and X.-H. Wang, "First-principles study of the electronic structure and optical properties of GaN(0001) surface," Acta Physica Sinica, vol. 61, no. 6, pp. 414-420, 2012.

[12] S. Zhang, J. Shi, M. Zhang, M. Yang, and J. Li, "First-principles investigation on optical properties of GaN and InGaN alloys," Journal of Physics D: Applied Physics, vol. 44, no. 49, pp. 495304495312, 2011.

[13] Z. Dridi, A. Lazreg, H. Rozale et al., "Electronic structure and magnetism of cubic $\mathrm{Ga}_{1-\mathrm{x}} \mathrm{Eu}_{\mathrm{x}} \mathrm{N}$ and $\mathrm{Al}_{1-\mathrm{x}} \mathrm{Eu}_{\mathrm{x}} \mathrm{N}$ using the LSDA+U approach," Computational Materials Science, vol. 48, no. 4, pp. 743-748, 2010.

[14] E. Yamaguchi and M. R. Junnarkar, "Effects of nitrogen vacancy on optical properties of nitride semiconductors," Journal of Crystal Growth, vol. 189-190, pp. 570-574, 1998.

[15] R. P. Davies, C. R. Abernathy, S. J. Pearton, D. P. Norton, M. P. Ivill, and F. Ren, "Review of recent advances in transition and lanthanide metal-doped $\mathrm{GaN}$ and $\mathrm{ZnO}$," Chemical Engineering Communications, vol. 196, no. 9, pp. 1030-1053, 2009.

[16] X. C. Sheng, The Spectrum and Optical Property of Semiconductor, Science Press, Beijing, China, 2003.

[17] K. Huang and R. Q. Han, Solid State Physics, Higher Education Press, Beijing, China, 1988.
[18] A. Sedhain, J. Li, J. Y. Lin, and H. X. Jiang, "Nature of deep center emissions in GaN," Applied Physics Letters, vol. 96, no. 15, pp. 1902-1907, 2010. 

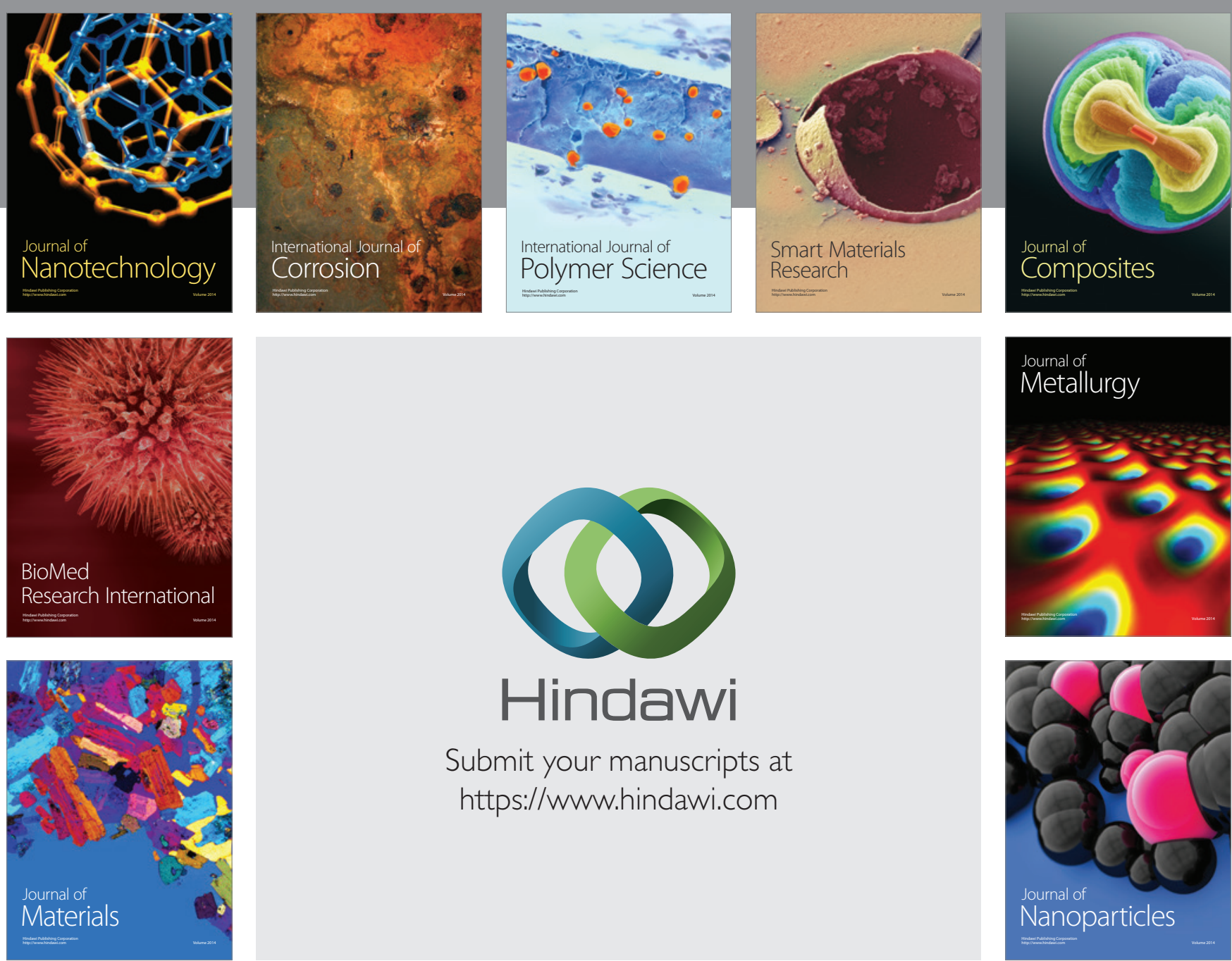

\section{Hindawi}

Submit your manuscripts at

https://www.hindawi.com

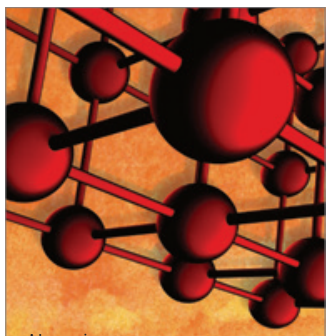

Materials Science and Engineering
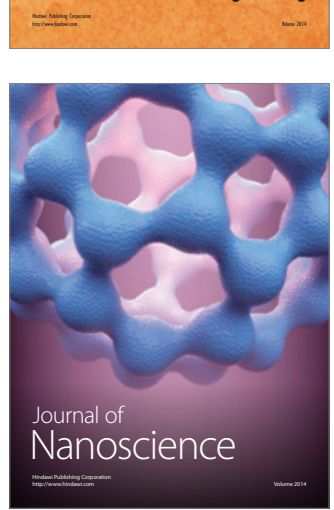
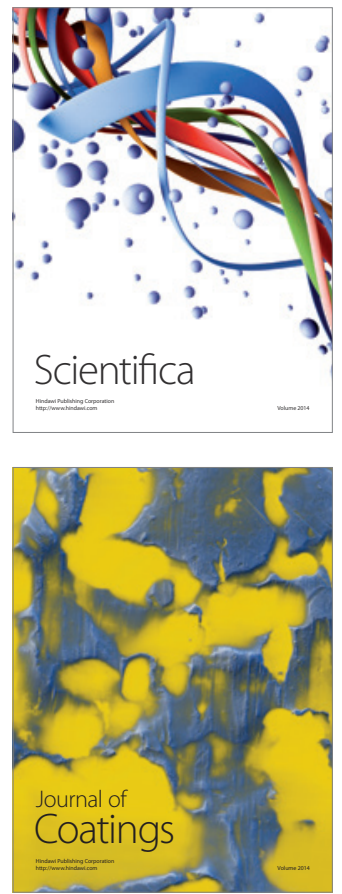
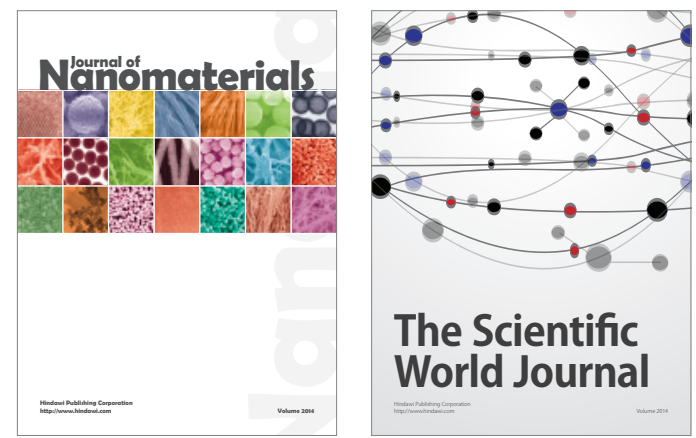

The Scientific World Journal
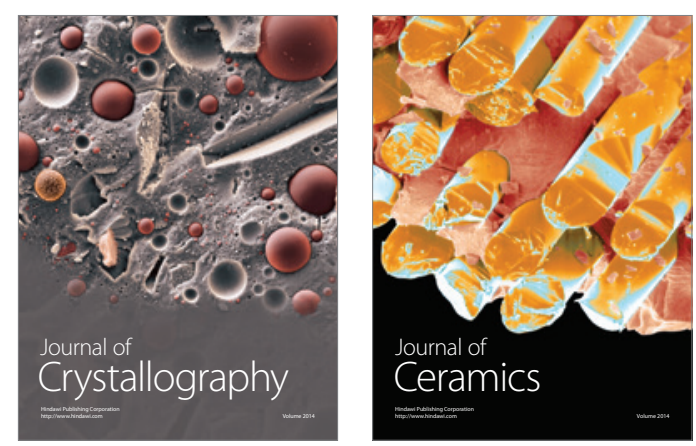
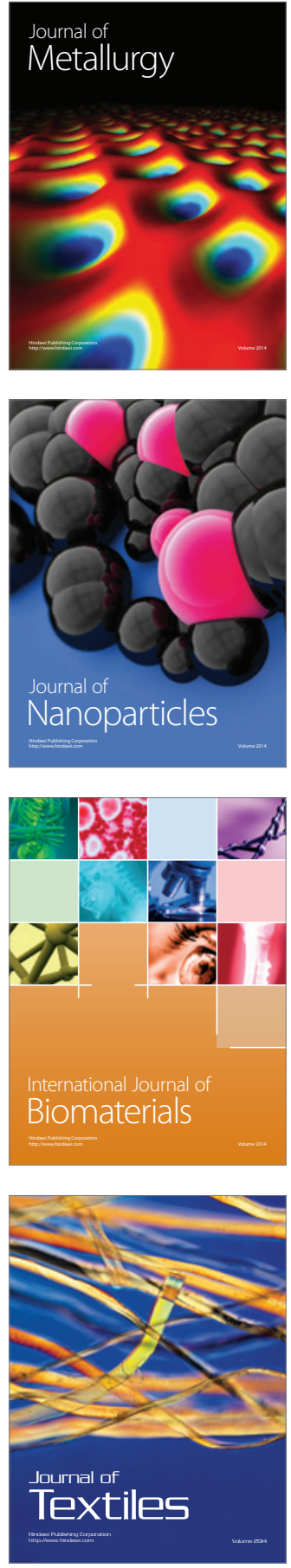